\title{
ON INFINITE DISJOINT COVERING SYSTEMS
}

\author{
AVIEZRI S. FRAENKEL AND R. JAMIE SIMPSON
}

(Communicated by William W. Adams)

\begin{abstract}
The structure of all infinite incongruent disjoint covering systems (IIDCS) whose moduli are divisible by no prime $>3$ is given. It is then shown that this structure characterizes the subset of IIDCS for which the greatest common factor of all the moduli is 1 , and the set of primes dividing the moduli is finite.
\end{abstract}

The integers, nonnegative integers, and positive integers are denoted by $\mathbb{Z}$, $\mathbb{Z}^{0}$, and $\mathbb{Z}^{+}$, respectively. By $b(\bmod a)$ we denote the arithmetic sequence of integers $\{n a+b: n \in \mathbb{Z}\}$, where $a \in \mathbb{Z}^{+}$is the modulus and $b \in \mathbb{Z}$ is the residue of $a$.

A system $\mathbf{S}=\left\{b_{i}\left(\bmod a_{i}\right): i \in I\right\}$ of arithmetic sequences that forms a partition of $\mathbb{Z}$ is called a disjoint covering system (abbreviated DCS). Here $I=\{1, \ldots, N\}$ is a finite index set, or $I=\{1,2, \ldots\}$ is countably infinite: $\mathrm{S}$ is said to be finite or infinite accordingly. A system $\mathrm{S}$ is called incongruent if $i \neq j \Rightarrow a_{i} \neq a_{j}$. Otherwise it is called congruent. It is well known that a DCS with $1<|I|<\infty$ cannot be incongruent. (See, e.g., Znám [7].) If $|I|=1$, then the DCS contains only the trivial arithmetic sequence with modulus 1 . Thus any incongruent DCS containing an arithmetic sequence with modulus $>1$ is necessarily infinite.

A major open problem is the characterization of DCS. In this note we shed some light on the nature of infinite incongruent DCS (abbreviated IIDCS).

The concept of the density $d$ of a subset of $\mathbb{Z}$ is central to our argument, so we start by collecting a few well-known or easy facts about it. A subset $S \subseteq \mathbb{Z}$ is periodic if there exists $t \in \mathbb{Z}^{+}$, called a period of $S$, such that $S+t=S$, where $S+t=\{s+t: s \in S\}$. The density of a periodic set $S$ is defined as $d(S)=\left|S \cap B_{t}\right| / t$, where $t$ is any period of $S$ and $B_{t}$ is any block of $t$ consecutive integers. This definition is consistent with the more general definition of asymptotic density which we do not require here. It is easy to verify that the family $\mathscr{P}$ of periodic subsets of $\mathbb{Z}$ is closed under finite unions, and $d\left(\bigcup_{i=1}^{N} S_{i}\right)=\sum_{i=1}^{N} d\left(S_{i}\right)$ whenever $S_{1}, \ldots, S_{N}$ are periodic and pairwise disjoint. This property, called finite additivity, does not extend to infinite unions: First, $\bigcup_{i=1}^{\infty} S_{i}$ need not be periodic even though each of the $S_{i}$ is periodic; second, even if $S_{i} \in \mathscr{P}(i=1,2, \ldots)$ are pairwise disjoint and

Received by the editors September 17, 1991 and, in revised form, January 13, 1992.

1991 Mathematics Subject Classification. Primary 05A17; Secondary 11 B83. 
$\bigcup_{i=1}^{\infty} S_{i} \in \mathscr{P}$, it need not be the case that $d\left(\bigcup_{i=1}^{\infty} S_{i}\right)=\sum_{i=1}^{\infty} d\left(S_{i}\right)$. What is true, however, under these assumptions is that $d\left(\bigcup_{i=1}^{\infty} S_{i}\right) \geq \sum_{i=1}^{\infty} d\left(S_{i}\right)$; this is seen by observing that $d\left(\bigcup_{i=1}^{\infty} S_{i}\right) \geq d\left(\bigcup_{i=1}^{N} S_{i}\right)=\sum_{i=1}^{N} d\left(S_{i}\right)$, and letting $N \rightarrow \infty$. We shall refer to this property of $d$ as superadditivity of the density.

Clearly $d(b(\bmod a))=1 / a$, which suggests that the reciprocals of the moduli of a DCS must satisfy certain conditions. For a multi-set of positive integers $A$, we denote $r(A)=\sum_{a \in A} 1 / a$. Superadditivity of the density implies that if $A$ is the multi-set of moduli of a DCS then $r(A) \leq 1$; we say that the DCS is saturated if $r(A)=1$. Otherwise it is unsaturated. Examples of both saturated and unsaturated IIDCS's were given in Stein [6]. We restrict attention here to saturated DCS's, because unsaturated ones are, in a sense, too easy to obtain. Finite additivity of the density guarantees that every finite DCS is saturated.

The following is an example of a saturated IIDCS:

$$
\begin{aligned}
S= & \left\{\frac{4^{i-1}-1}{3}\left(\bmod 2^{2 i-1}\right): 1 \leq i<\infty\right\} \\
& \cup\left\{3+10 \frac{4^{i-1}-1}{3}\left(\bmod 2^{2 i}\right): 1 \leq i<\infty\right\} .
\end{aligned}
$$

Stein [6] asked whether the moduli of every IIDCS are necessarily all powers of 2, as in (1). Krukenberg [4] answered this in the negative. See also Dewar [3]. Beebee [1] produced two IIDCS, each with moduli of the form $2^{\alpha} 3^{\beta}$, and commented that these systems can be varied to form infinitely many IIDCS, each with moduli of the form $2^{\alpha} 3^{\beta}$. He also asked the following (here slightly paraphrased) questions.

1. Is there a saturated IIDCS with smallest modulus larger than 4 ?

2. Is there a saturated IIDCS for which 1 is the greatest common divisor of the moduli?

3. Is there a (saturated) IIDCS with set of moduli

$$
\left\{2^{i} 3^{j}, 3^{j} 5^{k}, 2^{i} 5^{k}, 2^{i} 3^{j} 5^{k}: 1 \leq i, j, k<\infty\right\} ?
$$

Berger, Felzenbaum, Fraenkel, and Holzman [2] answered question 3 in the negative. As pointed out there, the same answer had also been given independently by Selfridge and by Simpson in unpublished manuscripts.

In this note we say something about the first two questions and the following additional question:

4. Is there a saturated IIDCS in which the set of distinct prime divisors of the moduli is infinite?

In Theorem 1 we show that the only saturated IIDCS with moduli divisible by no prime greater than 3 are IIDCS with moduli as in (1), which we call type I, and those called types II and III below, a special case of which are the two systems which appear explicitly in Beebee [1]. Using the methods of Theorem 1 and Corollary 1 of Simpson and Zeilberger [5], it can be shown that if the answer to question 4 is "yes", then there exists an IIDCS in which every prime divides some modulus. These results apply to finite covering systems, but the methods used can be adapted easily to saturated IIDCS as well. On the other hand we show in Theorem 2 that if the answers to Beebee's question 2 and our question 4 are both "no", then the only saturated IIDCS are those of types I, II, and III. This would imply that the answer to Beebee's question 1 is also "no". See Corollary 1 below. 
We now describe the three types of IIDCS with moduli of the form $2^{\alpha} 3^{\beta}$.

Type I has moduli $\left\{2^{\alpha}: 1 \leq \alpha<\infty\right\}$. For a possible assignment of residues see (1) above.

Type II with parameter $\gamma \in \mathbb{Z}^{0}$ has moduli

$$
\left\{2^{\alpha}: 1 \leq \alpha \leq \gamma\right\} \cup\left\{2^{\alpha}: \gamma+2 \leq \alpha<\infty\right\} \cup\left\{2^{\gamma+\alpha} 3^{\beta}: 1 \leq \alpha, \beta<\infty\right\} .
$$

If $\gamma=0$, then the set in the first pair of curly brackets is empty.

Type III with parameter $\gamma \in \mathbb{Z}^{0}$ has moduli

$$
\left\{2^{\alpha}: 1 \leq \alpha \leq \gamma\right\} \cup\left\{2^{\gamma+\alpha} 3^{\beta}: 0 \leq \alpha<\infty, 1 \leq \beta<\infty\right\} .
$$

The two systems given explicitly by Beebee are the special case $\gamma=0$ in both types II and III. However, for every $\gamma \in \mathbb{Z}^{0}$, the sets of moduli can be assigned appropriate residues to make them IIDCS. The technique for such assignments is implied in [1]. Type II with $\gamma=0$ gives a system with smallest modulus 4; in all other cases the smallest modulus is less than 4 . Further, the set of moduli of every IIDCS in the three types has a common factor greater than 1, namely 3 for type III with $\gamma=0$ and 2 in all other cases. Finally, it is easily verified that all IIDCS of the three types are saturated.

Prior to stating our first theorem, we note the following fact which follows easily from the Chinese Remainder Theorem.

Proposition 1. If $\left\{b_{i}\left(\bmod a_{i}\right): i \in I\right\}$ is a DCS, finite or infinite, congruent or incongruent, saturated or unsaturated, then so is $\left\{b_{i}+k\left(\bmod a_{i}\right): i \in I\right\}$ for every fixed integer constant $k$.

Theorem 1. Any saturated IIDCS with all moduli of the form $2^{\alpha} 3^{\beta}$ is one of the types I, II, or III.

Proof. If an IIDCS is not of type I, then at least one power of 2 does not occur in its set of moduli. Let $2^{\gamma+1}$ be the smallest power of 2 which is missing. The congruence classes with moduli $2,2^{2}, \ldots, 2^{\gamma}$ will cover all but one residue class modulo $2^{\gamma}$. By Proposition 1 we may suppose that this is $0\left(\bmod 2^{\gamma}\right)$. It is not hard to see that if $\gamma \geq 1$, then the residue class $2^{\gamma-1}\left(\bmod 2^{\gamma}\right)$ must belong to the IIDCS. The residue class $0\left(\bmod 2^{\gamma}\right)$ must be covered with classes of the form $b\left(\bmod 2^{\alpha} 3^{\beta}\right)$ with $b \equiv 0\left(\bmod 2^{\gamma}\right)$. We must have $\alpha \geq \gamma$, since if $\gamma \geq 1$, then $2^{\gamma-1}\left(\bmod 2^{\gamma}\right)$ and $b\left(\bmod 2^{\alpha} 3^{\beta}\right)$ would otherwise intersect by the Chinese Remainder Theorem, contradicting the disjointness of the system.

The moduli which are available to cover $0\left(\bmod 2^{\gamma}\right)$ may thus be partitioned into three disjoint sets:

$$
\begin{gathered}
A=\left\{2^{\gamma+\alpha}: 2 \leq \alpha<\infty\right\}, \quad B=\left\{2^{\gamma} 3^{\beta}: 1 \leq \beta<\infty\right\}, \\
C=\left\{2^{\gamma+\alpha} 3^{\beta}: 1 \leq \alpha, \beta<\infty\right\} .
\end{gathered}
$$

Since any arithmetic progression belonging to the IIDCS with modulus in $A \cup B \cup C$ has a residue $\equiv 0\left(\bmod 2^{\gamma}\right)$, it follows from the Chinese Remainder Theorem that moduli from both sets $A$ and $B$ cannot be in the same IIDCS. Thus moduli from $A$ but not $B$ can be used, or vice versa. Now $2^{-1}+2^{-2}+$ $\cdots+2^{-\gamma}=1-2^{-\gamma}$. It is easily checked that the sum of the reciprocals of all the elements of each $A, B$, and $C$ is $2^{-\gamma-1}$. Since our IIDCS is saturated, it follows that it must use all the elements of the sets $A$ and $C$, which gives a type II IIDCS with parameter $\gamma$, or all the elements of the sets $B$ and $C$, which gives a type III IIDCS with parameter $\gamma$. Q.E.D. 
A result equivalent to Theorem 1 is Theorem 7.2 of [4]. Because of its relative inaccessibility, we gave a proof here.

To prove the next theorem we need the following auxiliary result.

Lemma 1. Let $I \subseteq \mathbb{Z}^{+}$and let $S=\left\{b_{i}\left(\bmod a_{i}\right): i \in I\right\}$ be a DCS, finite or infinite, congruent or incongruent, saturated or unsaturated, and suppose that $m \mid a_{i}$ for all $i \in I$, where $m$ is a positive integer. For any $k \in\{0,1, \ldots, m-$ 1) define

$$
J_{k}=\left\{i \in I: b_{i} \equiv k(\bmod m)\right\} .
$$

Then $T_{k}=\left\{\left(b_{j}-k\right) / m\left(\bmod a_{j} / m\right): j \in J_{k}\right\}$ is also a DCS. Furthermore, if $S$ is incongruent or saturated, then $T_{k}$ is incongruent or saturated respectively.

Proof. Choose some $k \in\{0,1, \ldots, m-1\}$. Let $l$ be any integer. Since $S$ is a DCS, there is exactly one value $j \in I$ for which

$$
k+l m \equiv b_{j} \quad\left(\bmod a_{j}\right) .
$$

So actually $j \in J_{k}$, and

$$
l \equiv\left(b_{j}-k\right) / m \quad\left(\bmod a_{j} / m\right) .
$$

Thus $l$ belongs to $T_{k}$; and it cannot belong to more than one member of $T_{k}$ since $S$ is a DCS. Hence $T_{k}$ is a DCS. The construction implies that if $S$ is incongruent then so is $T_{k}$.

Since $T_{k}$ is a DCS, we have by superadditivity $\sum_{j \in J_{k}} m / a_{j} \leq 1$, so $\sum_{k=0}^{m-1} \sum_{j \in J_{k}} m / a_{j} \leq m$. If $S$ is saturated, the last inequality is an equality; hence, $\sum_{j \in J_{k}} m / a_{j}=1$ for all $k \in\{0, \ldots, m-1\}$, so $T_{k}$ is saturated. Q.E.D.

Theorem 2. If the answers to questions 2 and 4 are both "no", then the only saturated IIDCS are those of types I, II, and III.

Proof. Let $S=\left\{b_{i}\left(\bmod a_{i}\right): 1 \leq i<\infty\right\}$ be a saturated IIDCS. If the answer to question 2 is "no", then there is a prime $p_{i}$ which divides every modulus. If the answer to question 4 is "no", then there is a largest prime, say $p_{t}$, which divides some modulus of $S$. We will construct a saturated IIDCS in which $p_{t}$ divides every modulus. If $p_{i}=p_{t}$ we are done. Otherwise $S$ contains some arithmetic sequence of the form $b\left(\bmod n p_{t}^{\alpha}\right)$ where $p_{i} \mid n, p_{t} \nmid n$, and $\alpha \in \mathbb{Z}^{+}$. Let $k \in\left\{0,1, \ldots, p_{i}-1\right\}$ be such that $b \equiv k\left(\bmod p_{i}\right)$. We invoke Lemma 1 with $k$ and $m=p_{i}$, obtaining an incongruent saturated DCS which contains the arithmetic sequence $(b-k) / p_{i}\left(\bmod \left(n / p_{i}\right) p_{t}^{\alpha}\right)$. As remarked earlier, such a DCS must be infinite. We therefore have a saturated IIDCS containing the modulus $\left(n / p_{i}\right) p_{t}^{\alpha}$.

This procedure can now be repeated until we get a saturated IIDCS with $p_{t}$ as a common factor of the moduli. (It may be necessary to repeat the construction until the arithmetic progression becomes $b^{\prime}\left(\bmod p_{t}^{\alpha}\right)$ for some $b^{\prime}$, i.e., the only prime divisor of the modulus is $p_{t}$, in which case $p_{t}$ must clearly divide every modulus, or $p_{t}$ may appear as a common factor earlier.)

Thus we have a saturated IIDCS with common factor $p_{t}$. Suppose that the other primes dividing the moduli are $p_{1}, \ldots, p_{t-1}$, each of which is less than $p_{t}$. The moduli therefore come from the set

$$
\left\{p_{t} \prod_{i=1}^{t} p_{i}^{\alpha_{i}}: 0 \leq \alpha_{i}<\infty\right\} \text {. }
$$


The sum of the reciprocals of these numbers is

$$
\begin{aligned}
\frac{1}{p_{t}}\left(1+\frac{1}{p_{1}}+\frac{1}{p_{1}^{2}}+\cdots\right)\left(1+\frac{1}{p_{2}}+\frac{1}{p_{2}^{2}}+\cdots\right) \cdots\left(1+\frac{1}{p_{t}}+\frac{1}{p_{t}^{2}}+\cdots\right) \\
=\frac{1}{p_{t}} \prod_{i=1}^{t} \frac{p_{i}}{p_{i}-1} .
\end{aligned}
$$

This sum is 1 for $t=1, p_{1}=2$ and for $t=2, p_{1}=2, p_{2}=3$. In all other cases it is less than 1 . This is easily shown using induction on $t$. Since our IIDCS was assumed to be saturated, we must necessarily have $p_{t} \leq 3$. By Theorem 1 the IIDCS is then of type I, II, or III. Q.E.D.

Note that incongruency is not really necessary for this proof except at the very end, in invoking Theorem 1.

Corollary 1. If the answers to questions 2 and 4 are both "no", then also the answer to question 1 is "no".

Proof. All IIDCS of types I, II, and III have smallest modulus $\leq 4$. Q.E.D.

\section{REFERENCES}

1. J. Beebee, Examples of infinite, incongruent exact covers, Amer. Math. Monthly 95 (1988), 121-123.

2. M. A. Berger, A. Felzenbaum, A. S. Fraenkel, and R. Holzman, On infinite and finite covering systems, Amer. Math. Monthly 98 (1991), 739-742.

3. J. Dewar, On finite and infinite covering sets, Proc. Washington State Univ. Conf. on Number Theory (Washington State Univ., Pullman, WA) (J. H. Jordan and W. A. Webb, eds.), Washington State Univ. Press, Pullman, WA, 1971, pp. 201-206.

4. C. E. Krukenberg, Covering sets of the integers, Ph.D. Thesis, Univ. of Illinois, UrbanaChampaign, IL, 1971.

5. R. J. Simpson and D. Zeilberger, Necessary conditions for distinct covering systems with square-free moduli, Acta Arith. 59 (1991), 59-70.

6. S. K. Stein, Unions of arithmetic sequences, Math. Ann. 134 (1958), 289-294.

7. S. Znám, A survey of covering systems of congruences, Acta Math. Univ. Comenian. 40-41 (1982), 59-79.

Department of Applied Mathematics \& Computer Science, Weizmann Institute of SciENCE, 76100 REHOVOT, ISRAEL

Current address: Department of Mathematics, University of Pennsylvania, Philadelphia, Pennsylvania 19104-6395

E-mail address: fraenkel@thales.math.upenn.edu

School of Mathematics \& Statistics, Curtin University, Perth WA, 6001, Australia E-mail address: tsimpsonr@cc.curtin.edu.au 